\title{
Double polarized measurements with frozen spin target at MAMI
}

\section{Andreas Thomas ${ }^{1}$}

Institut für Kernphysik, Universität Mainz

J.-J.-Becherweg 45, D 55099Mainz, Germany

E-mail: thomandeuni-mainz.de

\section{For the A2-Collaboration}

The A2 Collaboration at the Mainz Microtron MAMI measures photon absorption cross sections using circularly and linearly polarized 'Bremsstrahlung' photons up to an energy of $\sim 1.5 \mathrm{GeV}$ and a polarized Frozen Spin Target. We use a $4 \pi$ detection system with the 'Crystal Ball' as central part. Our Frozen Spin Target provides both longitudinally and transversely polarized protons and deuterons.

In this paper the apparatus will be described. Double polarized experiments for the investigation of the nucleons excitation spectrum via meson production and our Compton program to measure the proton polarizabilities will be briefly discussed. 


\section{Experimental Apparatus}

\subsection{The A2 Photon Beam}

The MAMI accelerator with its source of polarised electrons, based on the photoeffect on a strained GaAs crystal, routinely delivers polarised beam with a maximum energy of $1604 \mathrm{MeV}$ and a degree of polarisation of approximately $85 \%$. The last accelerator stage, MAMI C, is realized as a Harmonic Double Sided Microtron (HDSM). Main features of this new machine concept are the four $90^{\circ}$ bending magnets and the two LINACs working on $2.45 \mathrm{GHz}$ and the first harmonic. Details of the machine can be found in reference [1].

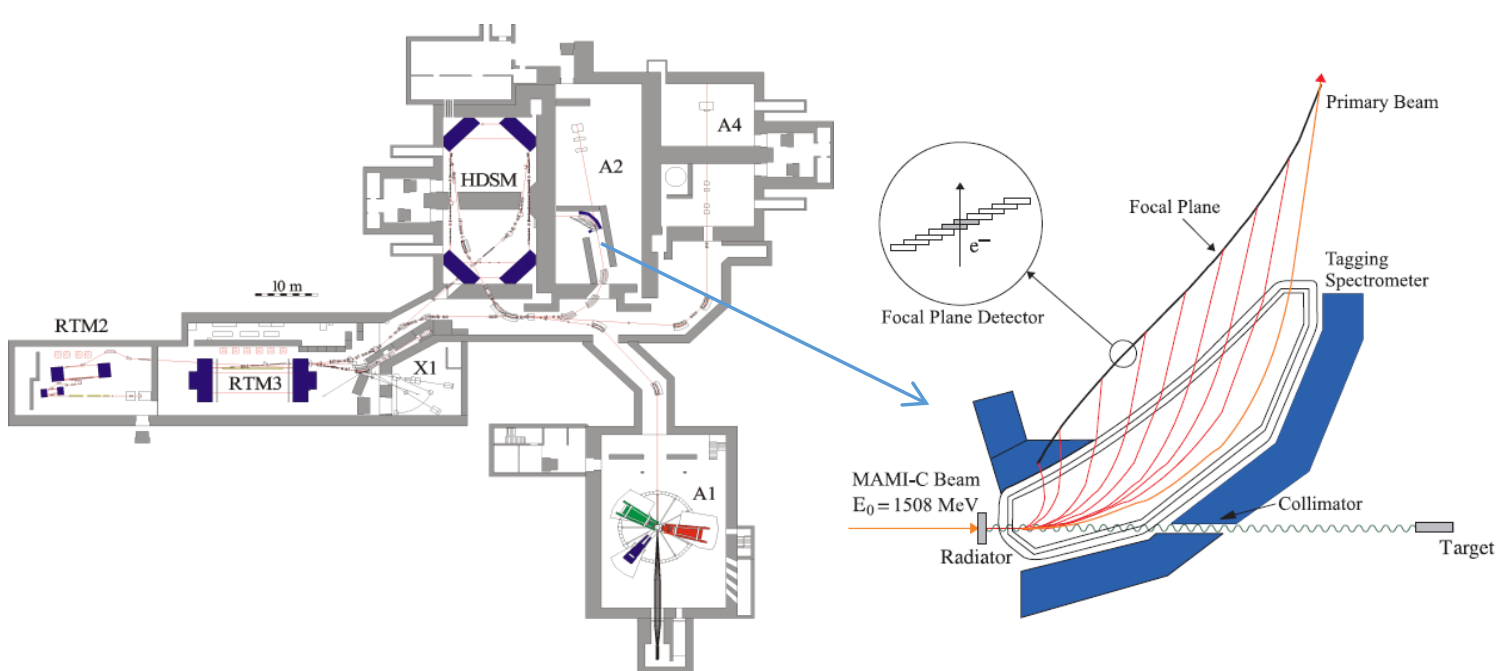

Figure 1. Floor plan (left side) of the MAMI accelerator with zoom in the Glasgow-Mainz tagging system (right side).

The A2-Glasgow-Mainz tagging facility [2] stands out due to its high photon intensity. The beam is derived from the production of Bremsstrahlung photons during the passage of the MAMI electron beam through a thin radiator. The resulting photons can be circularly polarised, with the application of a polarised electron beam, or linearly polarised, in the case of a crystalline radiator. The tagger focal plane is segmented into 352 scintillation detectors. Each counter can operate reliably to a rate of $1 \mathrm{MHz}$, giving high photon flux. The photons can be tagged in the momentum range from 4.7 to $93.0 \%$ of $\mathrm{E}_{0}$.

\subsection{The Crystal Ball detector setup}

The central detector system consists of the Crystal Ball calorimeter combined with a barrel of scintillation counters for particle identification and two coaxial multiwire proportional chambers for charged particle tracking. This central system provides position, energy and timing information for both charged and neutral particles in the region between $21^{\circ}$ and $159^{\circ}$ in the polar angle and over almost the full azimuthal range. At forward angles, less than $21^{\circ}$, reaction products are detected in the TAPS forward wall. The full, almost hermetic, detector system is shown schematically in figure 2 .

The full angular coverage of this detector system sets very rigorous condition for the construction of the polarized target. 


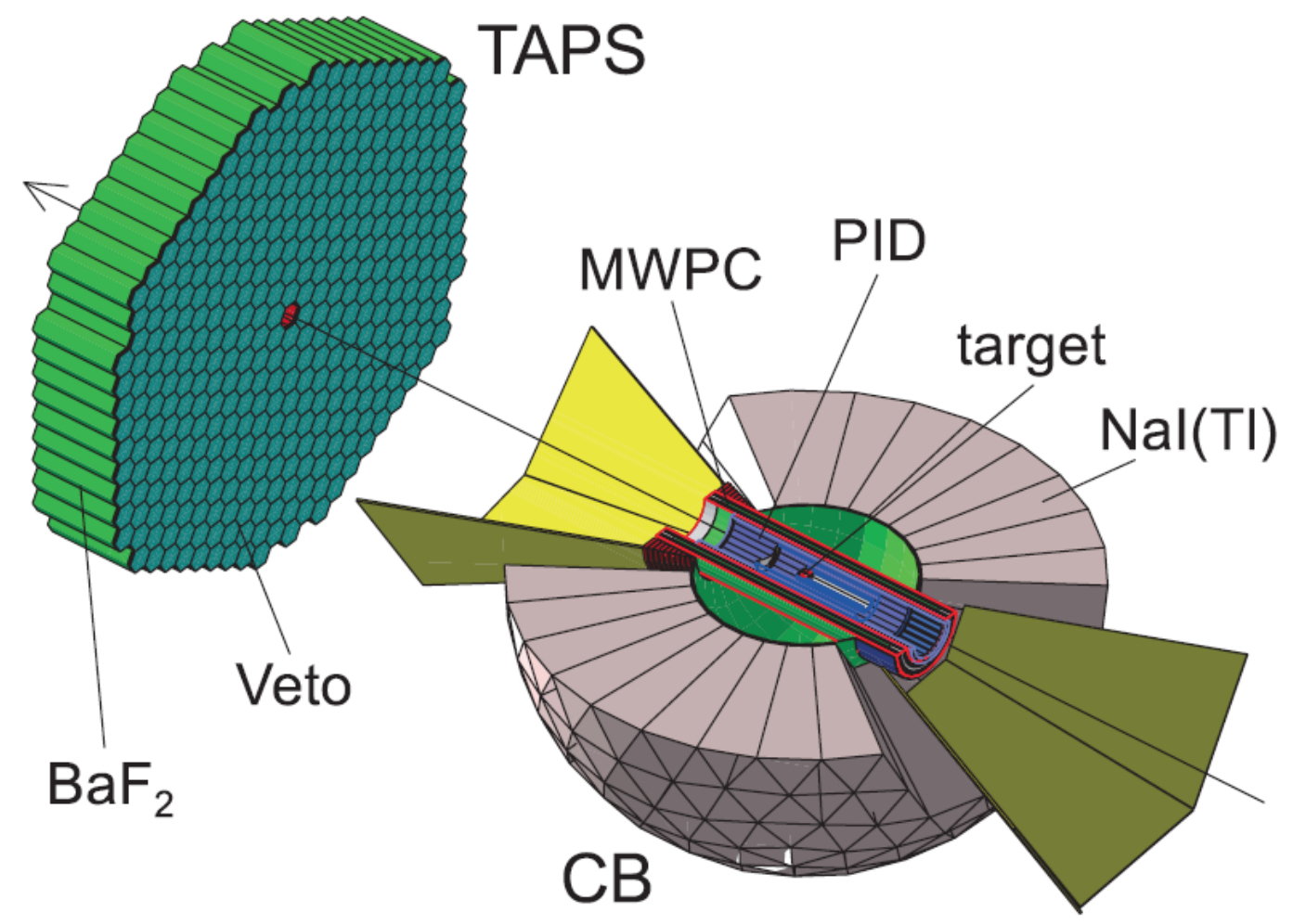

Figure 2. The Crystal Ball calorimeter, with cut-away section showing the inner detectors, and the TAPS forward wall.

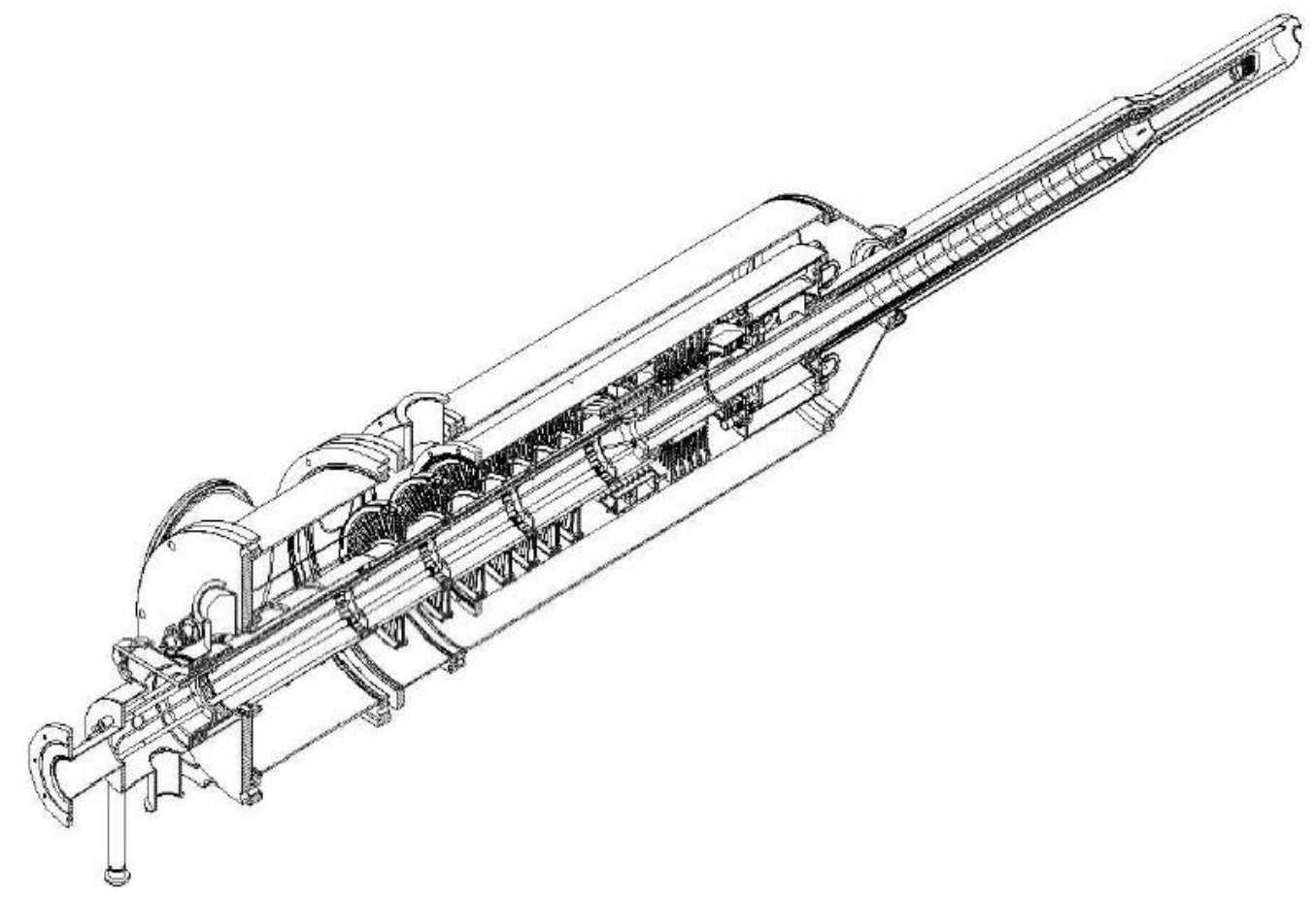

Figure 3. The ${ }^{3 / 4}$ helium dilution refrigerator. 


\subsection{The Polarized Target}

The new frozen spin target was designed to retain the high angular acceptance of the detector system. The main boundary condition for the outer diameter of the frozen spin target cryostat was the most inner particle identification detector with a diameter of 104 $\mathrm{mm}$. The internal holding coils had to be as thin as possible to allow particles to punch through. Longitudinal and transverse superconducting magnetic holding coils can be integrated to provide all directions of polarization.

The core of the frozen spin target for the Crystal Ball detector is a specially designed, large horizontal ${ }^{3} \mathrm{He} /{ }^{4} \mathrm{He}$ dilution refrigerator (see figure 3 ) that was built in cooperation with the Joint Institute for Nuclear Research (JINR) Dubna.

\section{Recent Double Polarized Measurements}

Several papers have been published in the last years [3-11] about our results concerning Baryon spectroscopy and meson photoproduction.

In addition, we have started an extended program to investigate un-polarized and polarized Compton scattering. Since the cross section is two orders of magnitude smaller compared to pion-production and the final state requires an open trigger the experiment is very challenging. These experiments will lead to a better determination of the scalar polarizabilities [12] and a first independent measurement of the spin polarizabilities [13].

Beside the measurements on a polarized frozen spin target with Butanol, where we use the polarized protons, we have also done a series of measurements with deuterated Butanol, in order to get access to the polarized neutron. In paper [9] the 'Helicitydependent cross sections and double-polarization observable $\mathrm{E}$ in $\eta$-photoproduction from quasi-free protons and neutrons' has been presented.
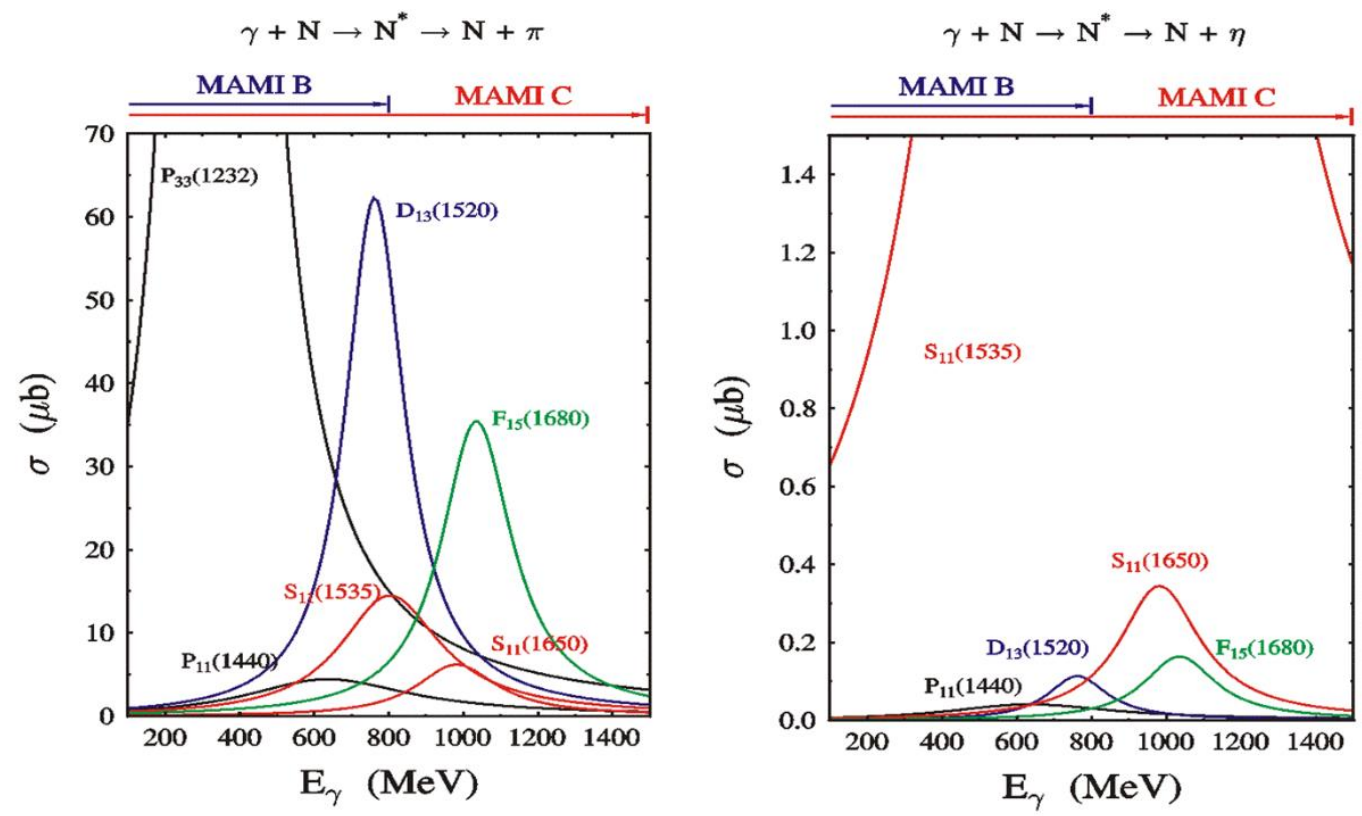

Figure 4. Breit-Wigner parametrisation of the resonances for $\pi$ - and $\eta$-photoproduction 
Figure 4 illustrates, how different the resonant contributions appear for the $\pi$ - and $\eta$ photoproduction. Beside the knowledge of the polarization observable the measurement of several reaction channels is the main tool to disentangle the broad and overlapping resonances. An additional information about the underlying physics can be achieved by using a proton and a neutron target and analyse and compare the result. In figures 5 and 6 from paper [9] we compare the helicity dependent differential cross-sections $\sigma_{1 / 2}$ and $\sigma 3 / 2$ for proton and neutron.
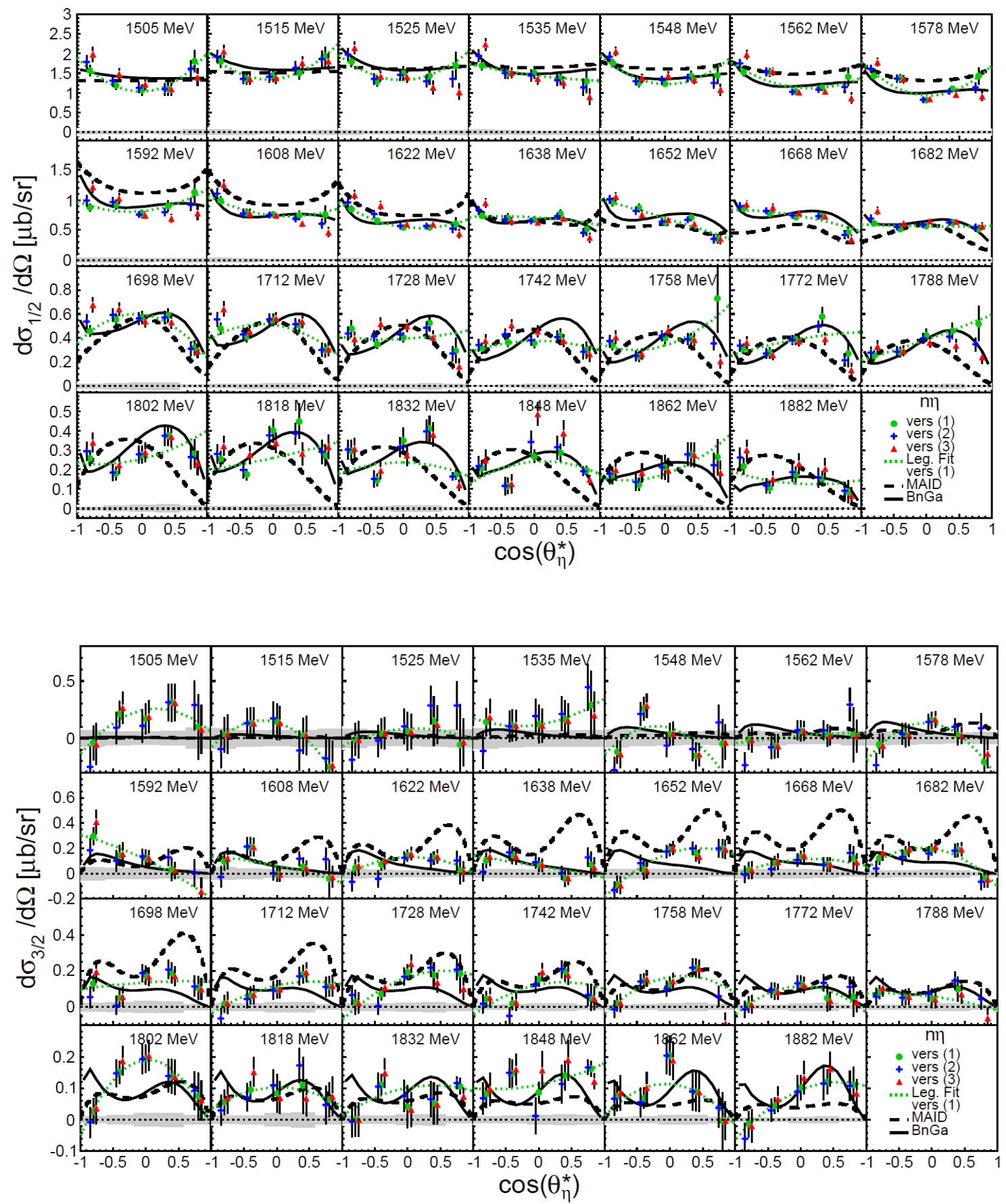

Figure 5. Helicity dependent cross sections for the $\eta$-photoproduction of protons [9]. 

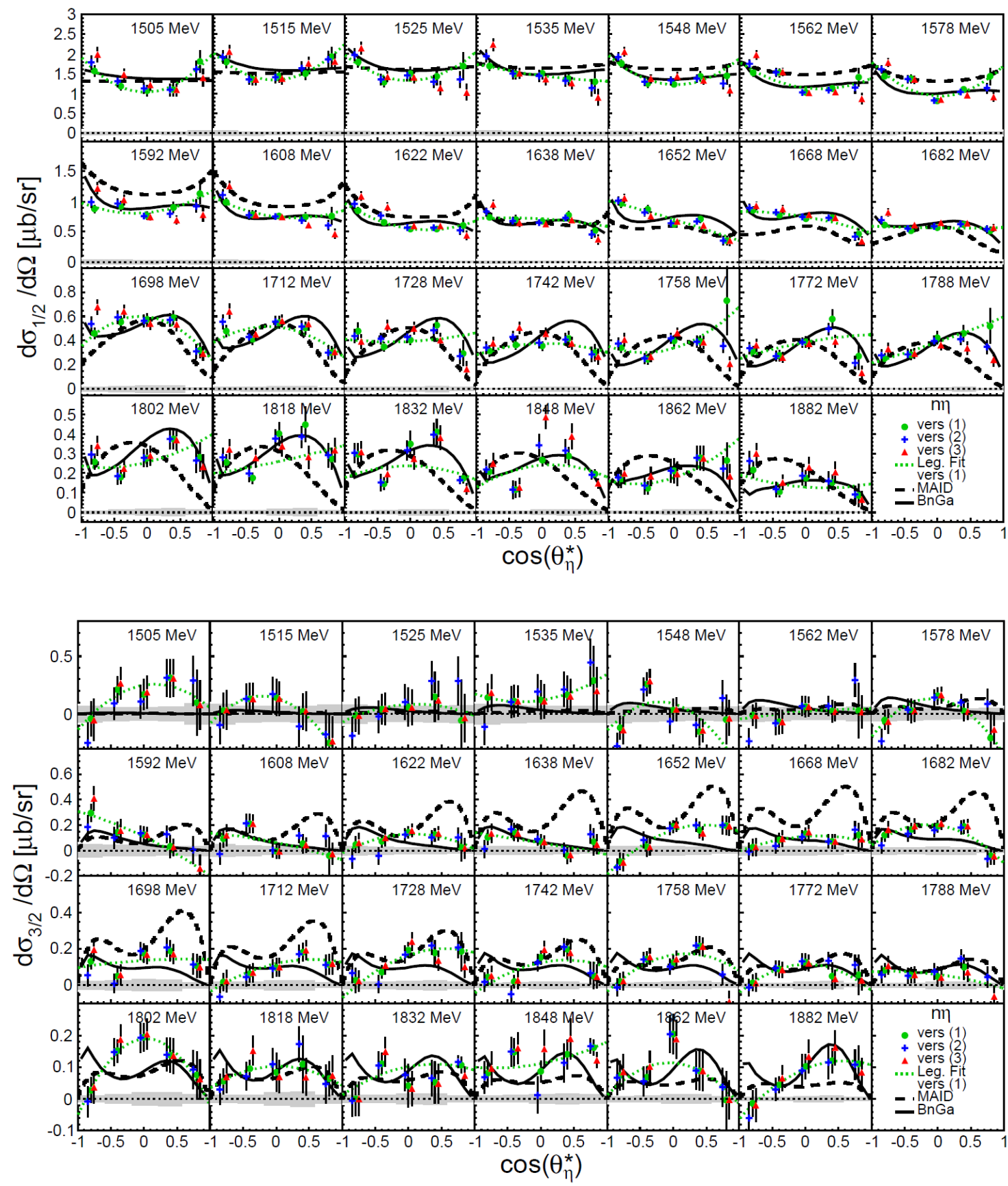

Figure 6. Helicity dependent cross sections for the $\eta$-photoproduction of neutrons [9].

These data confirm many previously known aspects of $\eta$-photoproduction and add key information to the interpretation in particular of the narrow structures seen in their excitation functions around invariant masses of $\mathrm{W} \approx 1.68 \mathrm{GeV}$. The most important one is that the narrow structure previously observed in the total cross section of the $\gamma \mathrm{n} \rightarrow \mathrm{n} \eta$ reaction appears only in the $\sigma 1 / 2$ part of the cross section and is thus almost certainly related to the S11 and/or P11 partial waves. At the same time the data 
with coincident protons show that the small dip observed in the total cross section of $\eta$ production from free protons at a similar energy can be assigned to structure in the $\sigma 3 / 2$ part of the reaction so that it is unlikely that both phenomena have the same cause.

\section{Acknowledgement}

The authors wish to acknowledge the excellent support of the accelerator group and operators of MAMI. This work was supported by the Deutsche Forschungsgemeinschaft (SFB1044), the European Community-Research Infrastructure Activity under the FP6 "Structuring the European Research Area" programme (Hadron Physics, Contract No. RII3- CT-2004-506078), Schweizerischer Nationalfonds (Contract Nos. 200020156983, 132799, 121781, 117601, 113511), the UK Science and Technology Facilities Council (STFC 57071/1, 50727/1), the US Department of Energy (O_ces of Science and Nuclear Physics, Award Nos. DE-FG02-99- ER41110, DEFG02-88ER40415, DEFG02-01-ER41194), and National Science Foundation (Grant Nos. PHY-1039130, IIA1358175), INFN (Italy), and NSERC (Canada). We thank the undergraduate students of Mount Allison University and The George Washington University for their assistance.

\section{References}

[1] A. Jankowiak, Eur. Phys. J. A 28, s01, 149 (2006)

[2] J.C. McGeorge et al., Eur. Phys. J. A 37, 129 (2008)

[3] P.Adlarson et al., Phys. Rev. C 92, 024617 (2015)

[4] M.Dieterle et al., Phys. Rev. Lett. 112, 142001 (2014)

[5] J.R.Annand et al., Phys. Rev. C 93, 055209 (2016)

[6] J.R.Annand et al., Phys. Rev. C 91, 055208 (2015)

[7] S.Schumann et al., Phys. Lett. B 750, 252 (2015)

[8] A. Käser et al., EPJ A 52, 272 (2016)

[9] L.Witthauer et al., Phys. Rev. Lett. 117, 132502 (2016)

[10] P. Adlarson et al, Phys. Rev. C 95, 035208 (2017)

[11] M.Dieterle et al., Phys. Lett. B, 523 (2017)

[12] P.P.Martel et al., Phys. Rev. Lett. 91, 112501 (2015)

[13] V. Sokhoyan et al., Eur. Phys. J. A 53, 14 (2017) 